\title{
SOME FIXED POINT RESULTS FOR $U V$ DECOMPOSITIONS OF COMPACT METRIC SPACES
}

\author{
JOHN COBB AND WILLIAM VOXMAN
}

\begin{abstract}
In this paper the preservation of the fixed point property under $U V$ decompositions is studied. It is shown that if $K$ is an $n$-dimensional complex with the fixed point property and $G$ is $U V^{n-1}$ decomposition of $K$, then $K / G$ also will have the fixed point property. Furthermore, if $X$ is a compact metric space with the fixed point property, and $G$ is a $U V^{n}$ decomposition of $X$ such that $X / G$ may be embedded in a suitably small Euclidian space, $R^{m}$, then $X / G$ retains the fixed point property.
\end{abstract}

Introduction. A space $X$ is said to have the fixed point property if each continuous function $f: X \rightarrow X$ leaves some point of $X$ fixed, i.e., there is a point $x \in X$ such that $f(x)=x$. In this paper it is shown that the fixed point property for compact metric spaces is preserved under suitable $U V$ decompositions. Examples and some applications of the main results are described. Proofs rely to a great extent on techniques utilized by Armentrout and Price in [1], and it would be helpful if the reader is familiar with this paper.

Notation and terminology. If $G$ is an upper semicontinuous decomposition of a topological space $X$, then $X / G$ will denote the associated decomposition space, and $P: X \rightarrow X / G$ the natural projection from $X$ onto $X / G$.

Suppose $X$ is a topological space, $M$ is a subset of $X$, and $n$ is a nonnegative integer. $M$ has property $n-U V$ if and only if for each open set $U$ containing $M$, there is an open set $V$ containing $M$ such that (1) $V \subset U$ and (2) each singular $n$-sphere in $V$ is homotopic to 0 in $U . M$ has property $U V^{n}$ if and only if for each nonnegative integer such that $i \leqq n, M$ has property $i-U V ; M$ has property $U V^{\omega}$ if for each nonnegative integer $k, M$ has property $k-U V$.

If $X$ is a topological space and $n$ is a nonnegative integer, the statement that $G$ is $U V^{n}$ decomposition of $X$ means that $G$ is an upper semicontinuous decomposition of $X$ into compact sets, each with property $U V^{n}$.

Received by the editors April 15, 1971.

AMS 1969 subject classifications. Primary 54B15; Secondary 54B25.

Key words and phrases. Simplicial complex, compact metric space, $U V$ decomposition, fixed point property.

(i.) American Mathematical Society 1972 
Suppose $X$ is a topological space. If $\mathscr{U}$ is a collection of subsets of $X$ and $A \subset X$, then the star of $A$ with respect to $\mathscr{U}$, denoted by $\operatorname{st}(A, \mathscr{U})$, is $\bigcup\{U: U \in \mathscr{U}$ and $U$ intersects $A\}$. Suppose $\mathscr{U}$ and $\mathscr{V}$ are collections of open subsets of $X$. Then $\mathscr{V}$ star refines $\mathscr{U}$ if and only if for each set $V$ of $\mathscr{V}$, there is a set $U$ of $\mathscr{U}$ such that $\operatorname{st}(V, \mathscr{V}) \subset U$. If $n$ is any nonnegative integer, then $\mathscr{V}$ star n-homotopy refines $\mathscr{U}$ if and only if for each set $V$ of $\mathscr{V}$, there is a set $U$ of $\mathscr{U}$ such that (1) $\operatorname{st}(V, \mathscr{V}) \subset U$ and (2) if $0 \leqq k \leqq n$, each singular $k$-sphere in $\operatorname{st}(V, \mathscr{V})$ is homotopic to 0 in $U$.

THEOREM 1. Suppose $K$ is an n-dimensional finite simplicial complex with the fixed point property and $G$ is a $U V^{n-1}$ upper semicontinuous decomposition of $K$. Then $K / G$ has the fixed point property.

Proof. Suppose $h$ is a map from $K / G$ into itself. To find a fixed point for $h$, we first show that for each $\varepsilon>0$, there exists a map $F: K \rightarrow K$ such that $d(P F, h P)<\varepsilon$, that is, $d(P F(y), h P(y))<\varepsilon$ for each $y$ in $K / G$ where $d$ is the metric of $K / G$. In order to apply the "lifting extension" of Price and Armentrout [1], we select an arbitrary point $x$ in $K$, and choose $x^{\prime} \in$ $P^{-1}[h P(x)]$. Let $f:\{x\} \rightarrow\left\{x^{\prime}\right\}$. Since $G$ is a $U V^{n-1}$ decomposition of the complex $K$, there exists by [1, Lemma 3.2, p. 435] an extension $F$ of $f$ to all of $K$ with the desired property, $d(P F, h P)<\varepsilon$. Thus, for each positive integer $n$, we may find a map $F_{n}: K \rightarrow K$ such that $d\left(P F_{n}, h P\right)<1 / n$. Let $x_{n}$ be a fixed point of $F_{n}$. Passing to a subsequence if necessary we may assume that the sequence $\left\{x_{n}\right\}$ converges to a point $z$. Then $P(z)$ will be a fixed point of $h$ since

$d(h P(z), P(z)) \leqq d\left(h P(z), h P\left(x_{n}\right)\right)+d\left(h P\left(x_{n}\right), P F_{n}\left(x_{n}\right)\right)+d\left(P F_{n}\left(x_{n}\right), P(z)\right)$ and the sum on the right side tends towards 0 for increasing values of $n$. Since $h$ was arbitrary it follows that $K / G$ has the fixed point property.

That $G$ cannot be an arbitrary decomposition of $K$ is seen from the following easy example.

EXAMPLE 1 . Let $K$ be the unit disk and $g$ the unit circle in $K$. Then $K$ has the fixed point property while $K / G$ does not, where $K / G$ is the decomposition space obtained by identifying $g$ to a point.

A fairly natural question would be the following. Suppose $G$ is a $U V^{\omega}$ decomposition of a finite simplicial complex $K$ such that $K / G$ has the fixed point property. Does this imply that $K$ also enjoys this property? The answer is negative as can be seen from the next example.

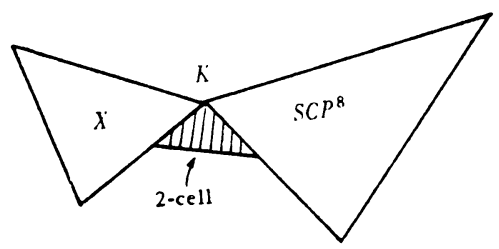


EXAmple 2. Let $K$ be the space described in Fig. 1(b) of [3, p. 21]. Here $X$ is the Lopez space $C P^{2} \cup S_{1} \times S_{2} \cup C P^{4}$ where $S_{1}$ and $S_{2}$ are 2spheres with $S_{1}$ identified with $C P^{1} \subset C P^{2}$ and $S_{2}$ identified with $C P^{1} \subset C P^{4}$. $S C P^{8}$ is the suspension of complex projective 8-space. To the wedge of $X$ and $S C P^{8}$ a 2-cell is attached. As indicated in [3], $K$ does not have the fixed point property. However, if we decompose the 2-cell into straight line segments (see above drawing), then the decomposition of $K$ whose nondegenerate elements consist of precisely these segments yields a decomposition space which is the wedge of $X$ and $S C P^{8}$ and, hence, has the fixed point property [3]. This decomposition is easily seen to be $U V^{\omega}$.

If certain dimension restrictions are placed on the decomposition space, then Theorem 1 may be extended to arbitrary compact metric spaces. We first establish a lemma (Lemma 2) which is itself of some general interest.

Lemma 1 (Armentrout and Price [1]). Suppose $X$ is a metric space, $n$ is a nonnegative integer, $G$ is a $U V^{n}$ decomposition of $X$, and $A$ is a subset of $X / G$. If $\mathscr{U}$ is an open covering of $A$, there exists an open covering $\mathscr{V}$ of $A$ such that $\left\{P^{-1}[V]: V \in \mathscr{V}\right\}$ star n-homotopy refines $\left\{P^{-1}[U]: U \in \mathscr{U}\right\}$.

Lemma 2. Suppose $X$ is a metric space, $m$ and $n$ are positive integers, $G$ is a $U V^{n}$ decomposition of $X$, and $X / G$ is a subset of (may be embedded in) $R^{m}$ where $m \leqq n+1$. Let $\varepsilon$ be a positive number. Then there exists an open set $U$ of $R^{m}$ which contains $X / G$ and a map $F: U \rightarrow X$ such that $d(P F(u), u)<\varepsilon$ for each $u \in U$.

ProOF. Let $\mathscr{W}$ be a finite open covering of $X / G$ by sets of diameter less than $\varepsilon / 2$. By repeated applications of Lemma 1 , we may obtain a sequence, $\mathscr{V}^{0}, \mathscr{V}^{1}, \cdots, \mathscr{V}^{m}$ of finite open coverings of $X / G$ such that $\left\{P^{-1}\left[V^{0}\right]: V^{0} \in \mathscr{V}^{0}\right\}$ star $n$-homotopy refines $\left\{P^{-1}[W]: W \in \mathscr{W}\right\}$, and for $1 \leqq i \leqq m$,

$$
\left\{P^{-1}\left[V^{i}\right]: V^{i} \in \mathscr{V}^{i}\right\}
$$

star $n$-homotopy refines $\left\{P^{-1}\left[V^{i-1}\right]: V^{i-1} \in \mathscr{V}^{i-1}\right\}$. Let $\delta$ be a Lebesgue number for $\mathscr{V}^{m}$ and $T$ be a triangulation of $R^{m}$ with mesh less than $\min \{\delta / 4, \varepsilon / 2\}$. Let $N=\{\sigma \in T: \sigma$ is an $m$-simplex and $\sigma \cap X / G \neq \varnothing\}$. For each $\sigma \in N$, choose a point $z_{\sigma} \in \sigma \cap X / G$, and with each vertex $v$ of a simplex in $N$, associate a point $y_{v}$ in $X / G$ in the following manner. If $v \in X / G$, then $y_{v}=v$, and if $v \notin X / G$, then $y_{v}$ is to be a point in $X / G$ of minimum distance from $v$. Note then that for every $\sigma \in N, \operatorname{diam}\left\{\left\{y_{v}: v\right.\right.$ a vertex of $\left.\left.\sigma\right\} \cup\left\{z_{\sigma}\right\}\right\}<\delta$. We now construct a function $F$ from $N^{*}=\bigcup\{\sigma: \sigma \in N\}$ into $X$ by first defining $F$ on the vertices, then extending $F$ to the 1 -simplices, then to the 2 -simplices, etc. If $v$ is a vertex of a simplex in $N$, we let $F(v) \in P^{-1}\left(y_{v}\right)$. Suppose $\tau$ is a 1 -simplex in some simplex of $N$ with vertices $v_{1}$ and $v_{2}$. It follows from our construction that there is a $V^{m} \in \mathscr{V}^{m}$ such that $y_{v_{1}}$ and $y_{v_{2}}$ are both contained in $V^{m}$ (since $d\left(x_{v_{1}}, y_{v_{2}}\right)<\delta$ ). Hence, $F\left(v_{1}\right)$ and $F\left(v_{2}\right)$ 
belong to $P^{-1}\left[V^{m}\right]$. Since $\left\{P^{-1}\left[V^{m}\right]: V^{n} \in \mathscr{V}^{-m}\right\}$ is a star $n$-homotopy refinement of $\left\{P^{-1}\left[V^{m-1}\right]: V^{m-1} \in \mathscr{V}^{m-1}\right\}$ there is a $V^{m-1} \in \mathscr{V}^{m-1}$ such that $F$ may be extended to a map of $\tau$ into $P^{-1}\left[V^{m-1}\right]$. Continuing in this manner (as described in [1, Lemma 3.2]) we may extend $F$ to all of $N^{*}$; furthermore, $F$ will have the property that for each $\sigma \in N$, there is a $V^{0} \in \mathscr{V}^{0}$ such that $F[\sigma] \subset P^{-1}\left[V^{0}\right]$. Observe also that $V^{0}$ may be chosen so that $z_{\sigma} \in V^{0}$, and of course, $V^{0} \subset W$ for some $W \in \mathscr{W}^{\text {r }}$.

Let $U=$ Interior $N^{*}$. To complete the proof we need to check that if $u \in U$, then $d(P F(u), u)<\varepsilon$. For $u \in U$ there exists a $\sigma \in N$ such that $u \in \sigma$. Then it is easily verified that

$$
d(P F(u), u) \leqq d\left(P F(u), z_{\sigma}\right)+d\left(z_{\sigma}, u\right)<\varepsilon / 2+\varepsilon / 2
$$

since $P F(u)$ and $z_{\sigma}$ both belong to some $W \in \mathscr{W}^{r}$.

THeOREM 2. Suppose $G$ is a $U V^{n}$ decomposition of a compact metric space $X$ such that $X / G$ may be embedded in $R^{m}$ for some $m \leqq n+1$. Then if $X$ has the fixed point property, so does $X / G$.

Proof. Suppose that $X / G$ does not have the fixed point property. Let $K: X / G \rightarrow X / G$ be a map which is fixed point free. Since $X / G$ is compact there is a positive number $\varepsilon$ such that $d(K(y), y) \geqq \varepsilon$ for each $y \in X / G$. By Lemma 2, we may find an open set $U$ in $R^{m}$ which contains $X / G$ and a map $F: U \rightarrow X$ such that $d(P F(u), u)<\varepsilon$ for each $u \in U$. Define $g=F \circ K \circ P$. Thus $g$ is a map from $X$ into $X$ and, hence, has a fixed point $z$. But $d(K P(z), P(z))<\varepsilon$ since $P(z)=P F(K P(z))$ and $d(P F(K P(z)), K P(z))<\varepsilon$. Since this contradicts the fact that $d\left(K\left(y^{j}\right), y\right) \geqq \varepsilon$ for each $y \in X / G, K$ must have a fixed point.

COROLlaRY 1. Suppose $G$ is a $U V^{\omega}$ decomposition of a compact metric space $X$ such that $X / G$ is finite dimensional. Then if $X$ has the fixed point property, so does $X / G$.

CoROllary 2. Suppose $G$ is a $U V^{n}$ decomposition of a compact metric space $X$ such that $\operatorname{dim} X / G \leqq n / 2$. Then if $X$ has the fixed point property, so does $X / G$.

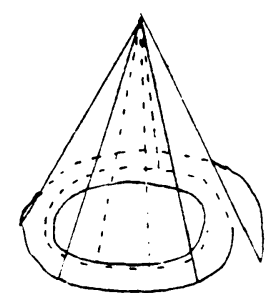

Figure 1

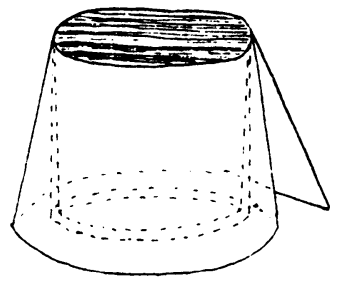

FIGURE 2

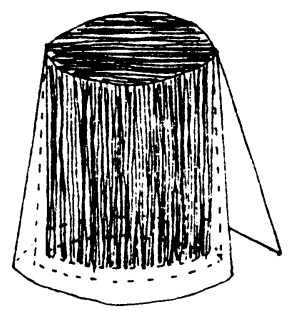

FIGURE 3 
Proof. Since $2(n / 2)+1 \leqq n+1, X / G$ may be embedded in $R^{n+1}$ and Theorem 2 applies.

As an application of Theorem 2, we consider the following spaces. Let $X$ be the cone over a circle with convergent spiral (Figure 1). Let $Y$ be the bottomless-can-with-skirt-with-lid-attached (Figure 2; see [2, p. 131]), and let $Z$ be the solid can-with-skirt (Figure 3).

$X$ does not have the fixed point property (see, for example, [2, p. 129]), and, hence, $Y$ cannot have the fixed point property; for if it did, the decomposition space obtained by identifying the lid to a point (a $U V^{\omega}$ decomposition) would also have the fixed point property-but this space is just $X$. It then follows that $Z$ fails to have the fixed point property (see also Knill [4]) since $Z$ retracts onto $Y$.

\section{BIBLIOGRAPHY}

1. S. Armentrout and T. Price, Decompositions into compact sets with UV properties, Trans. Amer. Math. Soc. 141 (1969), 433-442. MR 39 \#6307.

2. R. H. Bing, The elusive fixed point property, Amer. Math. Monthly 76 (1969), 119-132. MR 38 \#5201.

3. E. Fadell, Recent results in the fixed point theory of continuous maps, Bull. Amer. Math. Soc. 76 (1970), 10-29.

4. R. J. Knill, Cones, products and fixed points, Fund. Math. 60 (1967), 35-46. MR 35 \#2270.

Department of Mathematics, University of Idaho, Moscow, Idaho 83843 